\title{
La aplicación de la Teledetección en los cambios de la cobertura vegetal. Aplicación al tramo medio del río Jarama (Guadalajara)
}

\author{
García Rodríguez J.L. ${ }^{1}$, Bernabe Antonio V. ${ }^{2}$, Gardezi, A. ${ }^{1}$, Riesco J.A ${ }^{3}$, \\ Giménez Suárez M.C. ${ }^{1}$, García Robredo F. ${ }^{1}$ \\ ${ }^{1}$ Departamento de Ingeniería y Gestión Forestal y Ambiental. ETSI Montes, Forestales y Medio Natural. UPM. \\ ${ }^{2}$ Alumno del Programa de Hidrociencias. Colegio de Posgraduados Campus Montecillos, Estado de México. \\ ${ }^{3}$ Prosecar. Proyectos y Servicios Cartográficos, S.L. C7 La Bañeza, 43. 28035 Madrid. \\ *e-mail: josel.garcia@upm.es
}

\section{Resumen}

La ocupación del suelo y la transformación del medio natural es una de las principales preocupaciones ambientales. Los principales programas de inventario de los usos del suelo han incorporado con el paso del tiempo las imágenes de satélite, con el fin de hacer un seguimiento multitemporal. En este trabajo se pretende dar a conocer la evolución de los usos del suelo desde 1984 hasta 2013 en una cuenca en la que se ha trabajado mucho desde diferentes aspectos de la Hidrología Forestal y se pretende ver la gran diferencia con las tasas de erosión que se han obtenido en diferentes trabajos y estudios de investigación dentro de esta cuenca, con los modelos convencionales de erosión de la familia USLE, así como en los distintos enfoques de la Ordenación de la cuenca.

Palabras clave: Cobertura vegetal; imágenes Landsat; rio Jarama; uso del suelo. 


\section{Introducción}

Durante los últimos años existe un aumento sostenido en la utilización de imágenes satelitales de baja resolución para el monitoreo de la variación anual de la productividad de la vegetación. En escalas pequeñas de análisis, las bajas resoluciones espaciales son contrarrestadas por las altas resoluciones temporales (Carreiras et al., 2002; Tarwasky et al., 2008). El seguimiento de la productividad y el desempeño temporal y espacial de la vegetación son algunos de los principales desafíos en la investigación a partir de técnicas de teledetección, (Moulin et al., 2003; Chiesi et al., 2005; Lermitte et al., 2008). Mediante el uso de la teledetección es posible obtener información de las propiedades de la vegetación. Hasta cierto nivel es posible separar los tipos en regeneración de vegetación maduros (Foody et al., 1996). La reflectancia del infrarrojo cercano (TM4) proporciona información adecuada de la densidad de vegetación, en especial cuando se relaciona con área foliar y biomasa en hojas verdes y actividad fotosintética (Spanner et al., 1990; Baret y Guyot. 1991). En este principio se apoya la mayor parte de los denominados "índices de vegetación". En ellos se combinan la banda roja (R) e infrarroja (IR) del espectro.

El cambio de uso del suelo se ha convertido en un importante factor del cambio climático regional y global (Houghton et al., 1999, Chase et al., 2000, Claussen et

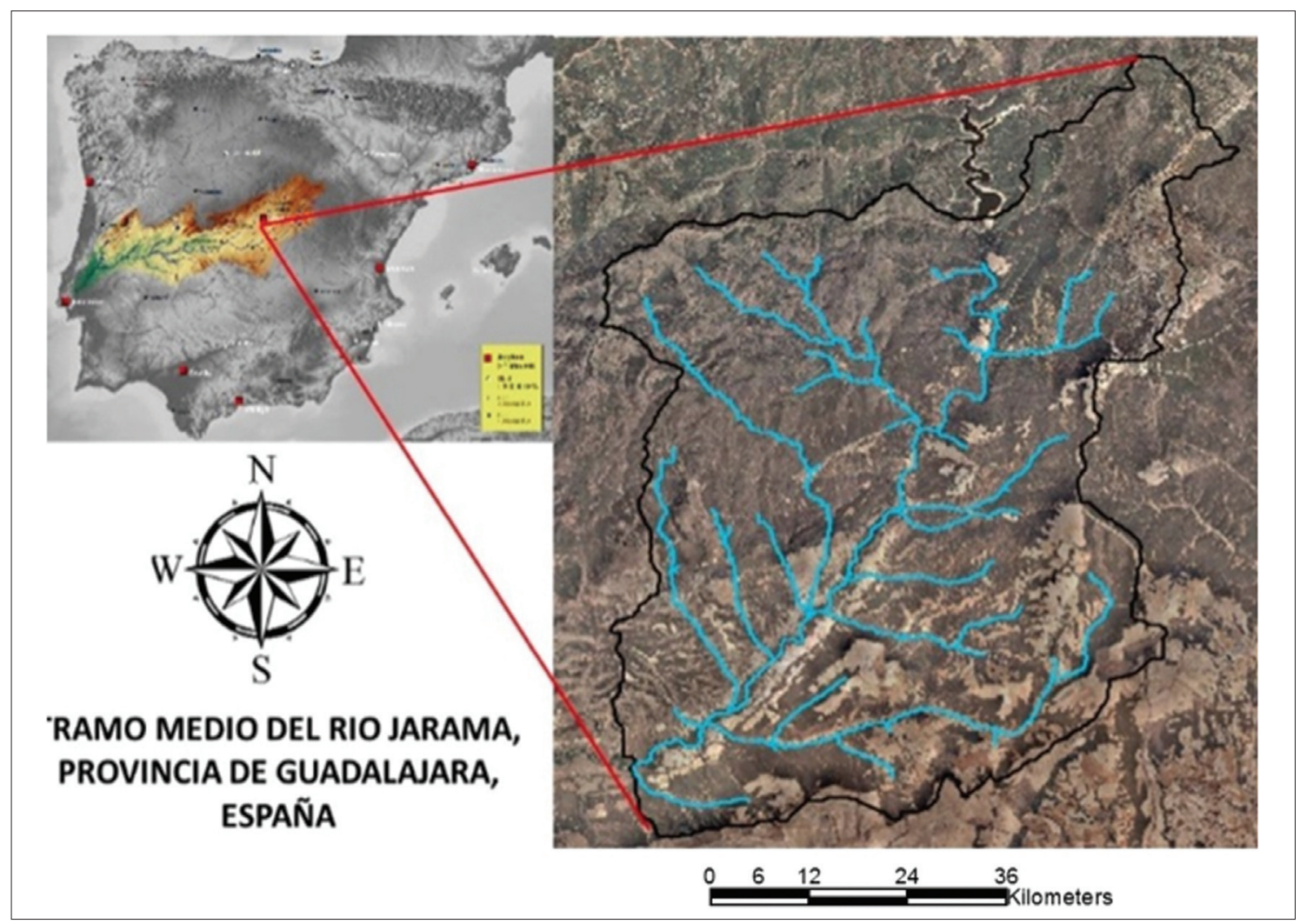

Figura 1. Localización del área de estudio del tramo medio del río Jarama. Guadalajara (España). Fuente: Google adaptado 
al., 2001, Defries et al., 2002, Brovkin et al., 2004); es considerado la primera causa de alteración del suelo (Matson et al., 1997, Lambin et al., 2001, Foley et al., 2005); y afecta, fuertemente, la capacidad de los sistemas biológicos para soportar y satisfacer las necesidades humanas (Vitousek et al., 1997, Foley et al., 2005). Tales cambios incrementan, a su vez, la vulnerabilidad de ecosistemas y personas frente a desastres naturales (Lambin et al., 2001). La pérdida, modificación y fragmentación de hábitats han provocado un fuerte declive de la biodiversidad a nivel mundial (Pimm y Raven 2000, Sala et al., 2000). El objetivo de este estudio es analizar la distribución espacial de las tendencias de uso de suelo y vegetación, mediante Imágenes Satelitales LANDSAT en el Tramo Medio del rio Jarama, esto con el propósito de realizar un análisis espacio temporal de la vegetación y cambios de usos de suelo durante el período 1984 - 2013 y de esta manera estimar la influencia de la cobertura vegetal.

\section{1 Área de estudio}

La cuenca se encuentra situada en la del río Tajo. Su localización geográfica se ubica entre $\operatorname{los} 40^{\circ} 50^{\prime}$ y $\operatorname{los} 41^{\circ} 03^{\prime}$ de latitud Norte y entre los $3^{\circ} 15^{\prime}$ y $3^{\circ} 25^{\prime}$ de longitud Oeste. Los límites naturales son: al Norte con cuenca alta del Jarama. Al Este, con la cuenca alta del río Sorbe, al Oeste con la cuenca del Lozoya y al Sur con la cuenca del Henares y tramo inferior del Jarama.

\section{Material y métodos}

El análisis de la dinámica de la cobertura y uso del suelo se realizó con base en dos imágenes satélite tipo Landsat TM y ETM, de agosto 1984 y 2013 (path 201, row 032). Los procesos desarrollados fueron los siguientes:

El análisis multitemporal post clasificatorio consistió en la transposición de dos mapas temáticos de fechas diferentes (producidos de manera independiente generalmente por clasificación de imágenes) con el fin de generar una matriz de detección de cambio que sintetiza los cambios del tiempo t al tiempo $\boldsymbol{t}+\boldsymbol{n}$ de las distintas categorías representadas en los mapas (Dobson et al., 1995; Jensen et al., 1998; Mas 1999; Ramsey III et al., 2001; Berlanga-Robles y Ruiz-Luna 2002). El análisis se realizó en seis etapas:

1. Edición de escenas multiespectrales de cada año.

2. Producción de mapas temáticos por la clasificación supervisada de las escenas y la digitalización de datos auxiliares.

3. Evaluación de la exactitud de los mapas temáticos.

4. Corrección geométrica y radiométrica de los mapas temáticos con base a los parámetros espaciales del mapa de 1984.

5. Transposición de mapas temáticos y generación de matrices de cambio.

6. Estimación de indicadores de cambio a nivel de clase. 


\section{Análisis de la información y cuantificación de los cambios}

Las coberturas vectoriales resultantes de los mapas temáticos fueron introducidas a un SIG para su correspondiente análisis espacial y temporal, cuyos resultados se analizaron estadísticamente a partir de los cambios detectados en la cobertura y uso de la tierra. El cambio de uso del suelo fue cuantificado usando una matriz de transición que consiste en una tabla simétrica de doble entrada en cuyos ejes se presentan las coberturas del suelo y en cada celda la superficie de las áreas que experimentaron cambios entre los años 1984 y 2013. Las celdas situadas en la diagonal de la matriz contienen la superficie de aquellas áreas que no experimentaron cambios durante el período (López et al., 2001, Aguayo et al., 2007). La matriz de transición fue construida a partir de la superposición y del algebra de mapas en ArcGIS. Las tasas de cambio fueron calculadas utilizando la siguiente fórmula:

$$
P=\frac{100}{t_{2}-t_{1}} \ln \frac{S_{2}}{S_{1}}
$$

Donde, $S_{1}$ y $S_{2}$, son las superficies en los tiempos t y ta, respectivamente, y $P$, es el porcentaje de cambio por año (FAO 1996, Echeverría et al., 2006).

\section{Resultados}

\section{Pastizales permanentes}

El avance de pastizales permanentes, que registraron el valor de ganancia más alto para el período (Tabla 2), explica gran parte de los cambios en las últimas tres

A)

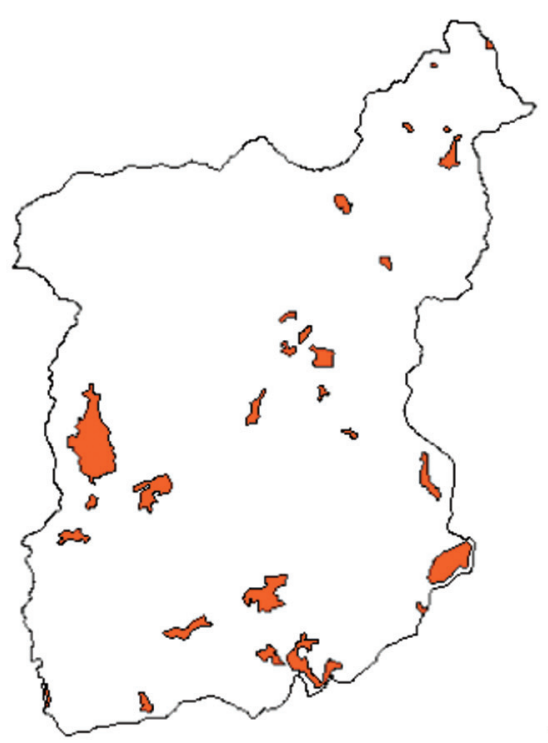

B)

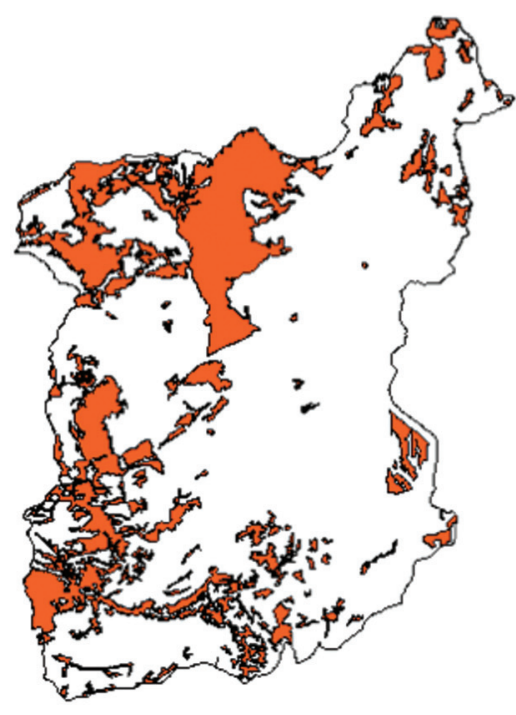

Figura 2. Mapa de cambio de los pastizales permanente donde se aprecia el aumento de casi seis veces durante el año 1984 (A) y el año 2013 (B). 


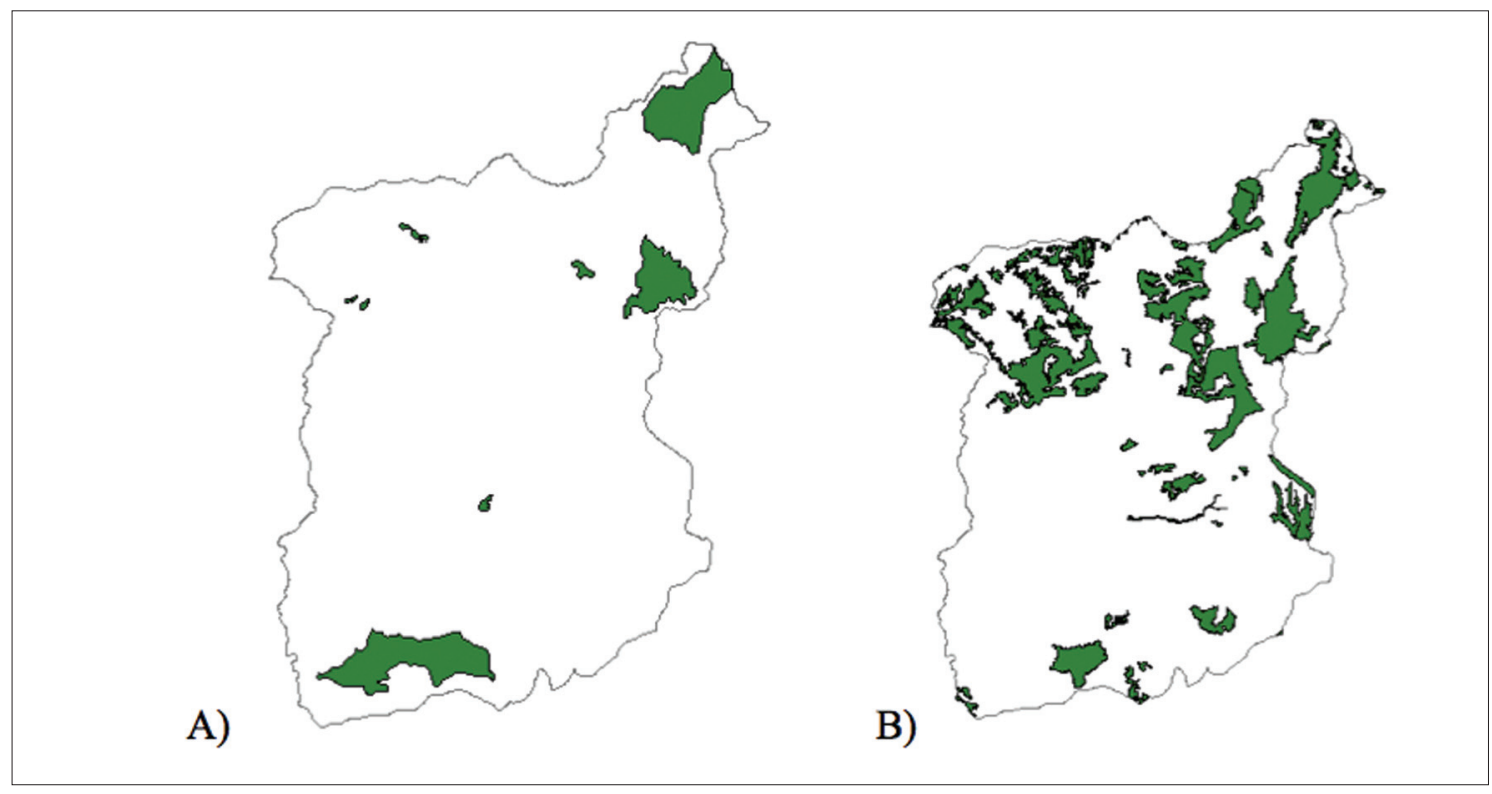

Figura 3. Mapa de cambio del arbolado con Fcc 20 - $70 \%$, donde se aprecia el aumento de 2.5 veces entre el año 1984 (A) y el año 2013 (B).

décadas. El $36.6 \%$ del incremento neto ocurrió sobre improductivo, el $25.2 \%$ ocupando áreas cubiertas por arbustos y matorral, $22.3 \%$ por cultivos herbáceos de secano, el $21,2 \%$ por arbolado con cabida, Fcc $>70 \%$, el $18.4 \%$ erial a pastos Fcc $<20 \%$, el $17.0 \%$ por cultivos arbóreos y viñedos de secano y solo un $11.4 \%$ por arbolado con cabida, Fcc $20-70 \%$.

A)

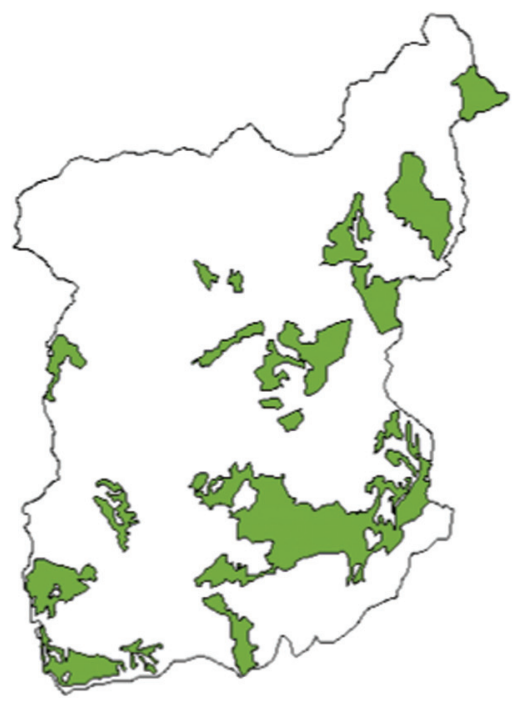

B)

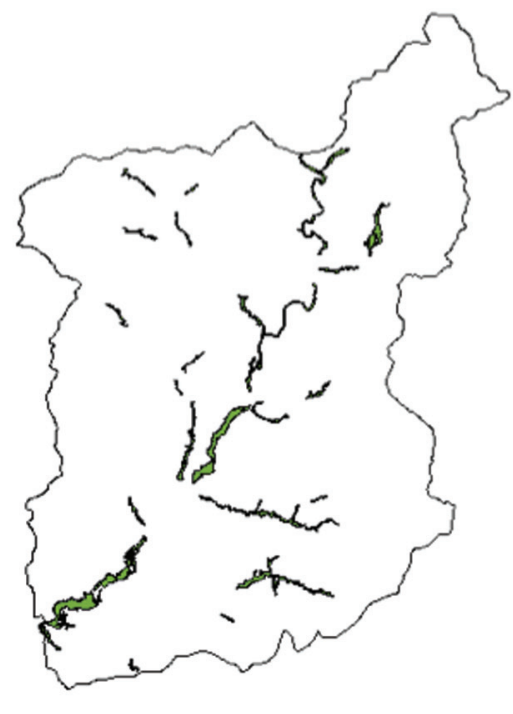

Figura 4. Mapa de cambio de erial a pastos $\mathrm{Fcc}<20 \%$ donde se aprecia una disminución del $88.3 \%$ entre el año 1984 (A) y el año 2013 (B). 
A)

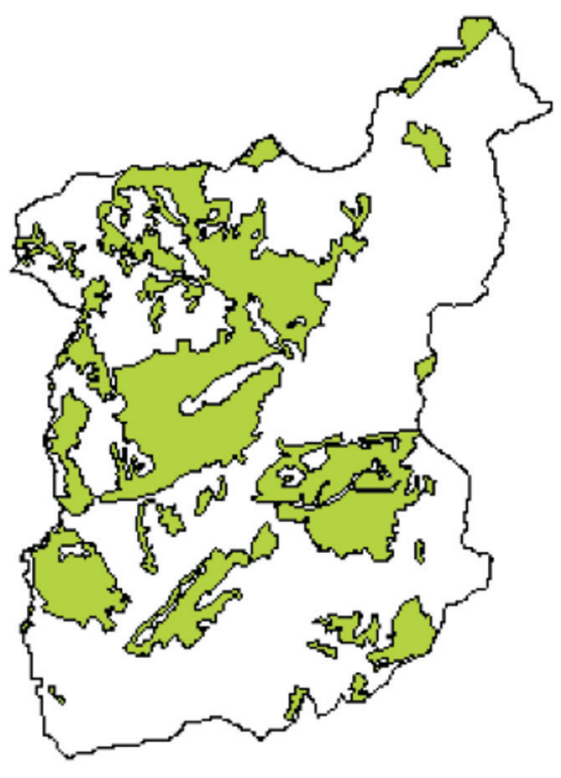

B)

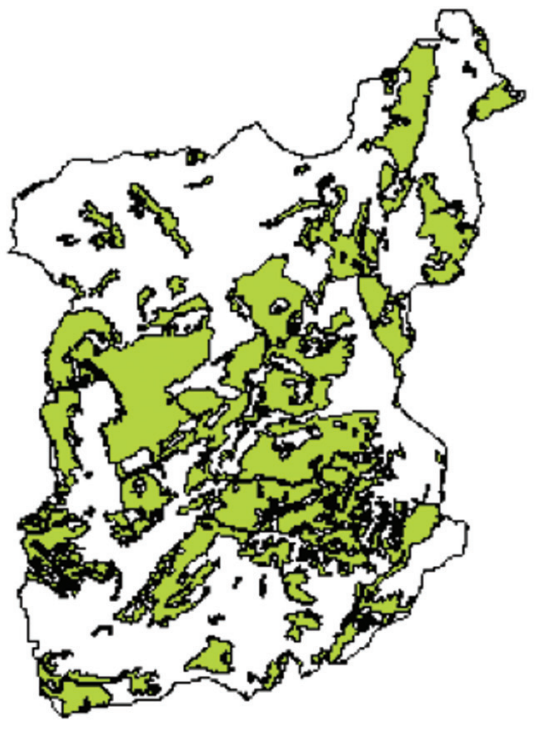

Figura 5. Mapa de cambio de Arbustos y matorral donde se aprecia un incremento del $12.6 \%$, que representan 901 ha del año 1984 (A) al año 2013 (B).

\section{Arbolado con cabida Fcc 20 - 70 \%.}

Durante el período de análisis esta cobertura registró una ganancia del $152.2 \%$ respecto a la superficie ocupada el año 1984. Esta dinámica generó una tasa de cambio anual positiva del $3.2 \%$ (Tabla 2). Las ganancias adquiridas fueron por efecto de pérdidas de arbolado con cabida Fcc $>70 \%$ con un $32.7 \%$, el improductivo con $13.3 \%$, cultivos herbáceos de secano $10.4 \%$, erial a pastos Fcc $<20 \%$ con $9.4 \%$, arbustos y matorral $9.1 \%$, pastizales permanentes con $4.7 \%$ y solo un $1 \%$ en pérdidas de cultivos arbóreos y viñedos de secano.

\section{Erial a pastos Fcc $<0.2$}

Entre 1984 y 2013 fue posible cuantificar una pérdida neta de 3843.20 hectáreas erial a pastos Fcc $<20 \%$ que equivale al $88.3 \%$ del total existente en el año 1984. Durante este período fue reemplazado a una tasa del $-7.4 \%$ anual (Tabla 2 ). El $4.8 \%$ de las pérdidas fue producto de la sustitución por el improductivo; el $3.9 \%$ pasó a la clase de cultivos herbáceos de secano; el $3.8 \%$ lo sustituyo los cultivos de regadío, $2.5 \%$ por arbolado con cabida Fcc $>70 \%, 2.6$ por cultivos arbóreos y viñedos de secano, $1.4 \%$ por pastizales permanentes, $0.7 \%$ por arbustos y matorral, $0.3 \%$ por arbolado con cabida Fcc 20 - 70 \% y solo el $1.7 \%$ se mantuvo constante durante el periodo.

\section{Arbolado con cabida Fcc $>70 \%$}

Durante el periodo de estudio esta clase tuvo una pérdida de 3012 ha, con un porcentaje de cambio por año de -4.8. Fueron reemplazados por arbolado con cabida Fcc $20-70 \%$ con un $17.1 \%$, seguida de todas las demás coberturas con excepción de los cultivos de regadío en un rango de 0.8 y $2.1 \%$. 


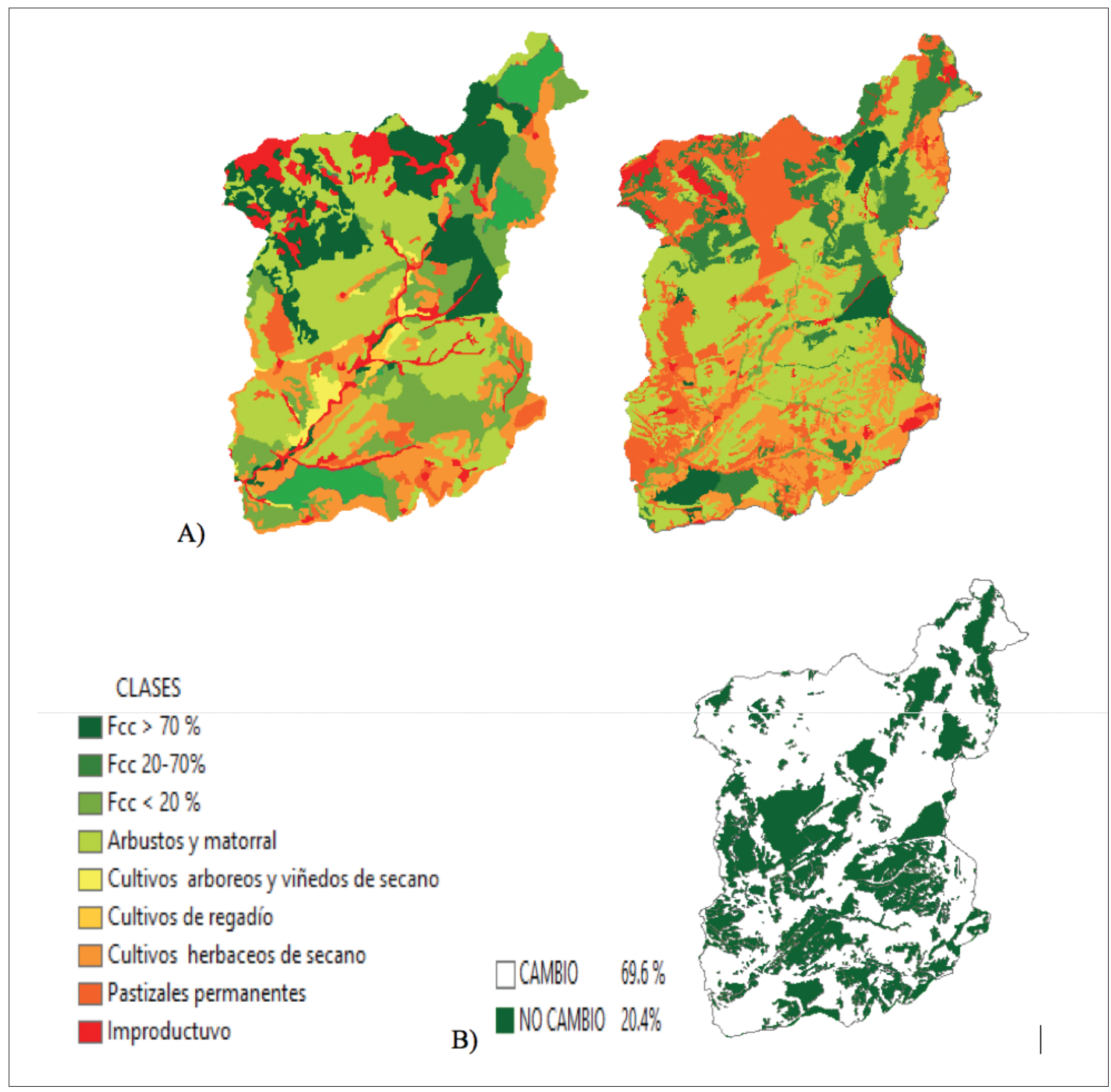

Figura 6. A) Mapa de uso de suelo y cobertura vegetal 1984 (izquierdo) y 2013 (derecha) con Imagen LANDSAT; donde se aprecia que los pastizales permanentes se multiplicaron en casi seis veces y donde el improductivo se refieren a zonas de erosión y cárcavas. B) Mapa de detección de cambios, se puede apreciar que los cambios afectaron a un 60\% de la superficie de la cuenca entre los años 1984 y 2013.

\section{Arbustos y matorral}

Sufrieron cambios positivos de un 30.17 a un $36.52 \%$ durante las últimas tres décadas, con un balance neto de $12.6 \%$ y un porcentaje de cambio por año de 0.4 (Tabla 1). Las ganancias se obtuvieron de erial a pastos Fcc $<20 \%$, cultivos arbóreos y viñedos de secano y cultivos de regadío perdiendo $50.4,39.3$ y $31.7 \%$ de su cobertura respectivamente (Tabla 2).

\section{Cultivos herbáceos de secano}

Esta clase de cobertura tiene un incremento de 666 hectáreas durante el periodo de estudio, con un balance neto de $21.7 \%$ y un porcentaje de cambio por año de 
0.7. Sus ganancias provienen del abandono de los cultivos de regadío con un $64.5 \%$, cultivo arbóreos y viñedos de secano con un $36.7 \%$, pastizales permanentes con un $22.4 \%$, erial a pastos Fcc $<20 \%$ con un $17.7 \%$, arbustos y matorral con $9.2 \%$, improductivo con un $8.6 \%$, arbolado con cabida Fcc 20 - $70 \%$ con un $3.8 \%$ y arbolado con cabida Fcc $>70 \%$ con un $1.1 \%$ (Tabla 1 ).

\section{Cultivos arbóreos y viñedos de secano}

Según el análisis de la Tab. 1, los resultados arrojan una pérdida de 431 hectáreas, que corresponden a un $97.9 \%$ de balance neto negativo y un cambio por año negativo porcentual de 13.3. Estas pérdidas cambiaron a los cultivos herbáceos de secano, pastizales permanentes y arbolado con cabida Fcc 20 - 70 \%, con un 54.4, 22.3 y $10.4 \%$ respectivamente.

\section{Cultivos de regadío}

Tienen una pérdida de 100 hectáreas, equivalente al $92.8 \%$ de balance neto negativo y un porcentaje de cambio por año de 9.1\% negativo (Tabla 1). Estas perdida se manifestaron por el abandono de esta cobertura transformadas en cultivos herbáceos de secano con un $64.5 \%$ y arbustos y matorral con un $31.8 \%$.

\section{Improductivo}

Obtuvo un efecto de balance neto negativo del $65.7 \%$ durante el periodo y un porcentaje de cambio por año negativo de 3.7 (Tabla 1). Los espacios ocupados por esta cobertura fueron por pastizales permanentes $(5.0 \%)$, cultivos herbáceos de secano (3.7\%), arbolado con cabida Fcc $>70 \%$ (2.3\%), arbustos y matorral $(2.2 \%)$, erial a pastos Fcc $<20 \%(1.6 \%)$ y arbolado con cabida Fcc $20-70 \%(1.2 \%)$.

Tabla 1. Uso de suelo y vegetación para los años 1984 y 2013, en superficie y balance neto y porcentaje de cambio por año.

\begin{tabular}{|c|c|c|c|c|c|c|c|}
\hline \multirow{2}{*}{$\begin{array}{l}\text { Uso y cubierta } \\
\text { vegetal }\end{array}$} & \multicolumn{2}{|c|}{ Superficie 1984} & \multicolumn{2}{|c|}{ Superficie 2013} & \multicolumn{2}{|c|}{ Balance neto } & \multirow{2}{*}{ Porcentaje } \\
\hline & ha & $\%$ & ha & $\%$ & ha & $\%$ & \\
\hline Improductivo & 1887 & 8.2 & 647 & 2.8 & -1240 & -65.7 & -3.7 \\
\hline Cultivos de regadío & 108 & 0.5 & 8 & 0.0 & -100 & -92.8 & -9.1 \\
\hline $\begin{array}{l}\text { Cultivos arbóreos } \\
\text { de secano3 }\end{array}$ & 441 & 1.9 & 9 & 0.0 & -431 & -97.9 & -13.3 \\
\hline $\begin{array}{c}\text { Cultivos herbáceos } \\
\text { de secano3 }\end{array}$ & 064 & 13.3 & 3731 & 16.2 & 666 & 21.7 & 0.7 \\
\hline Arbustos y matorral & 7128 & 31.0 & 8029 & 34.9 & 901 & 12.6 & 0.4 \\
\hline Pastizales permanentes & 935 & 4.1 & 5358 & 23.3 & 4423 & 473.1 & 6.0 \\
\hline Arbolado Fcc $<20 \%$ & 3944 & 17.1 & 461 & 2.0 & -3483 & -88.3 & -7.4 \\
\hline Arbolado Fcc: $20-70 \%$ & 1495 & 6.5 & 3770 & 16.4 & 2276 & 152.2 & 3.2 \\
\hline Arbolado Fcc $>70 \%$ & 4026 & 17.5 & 1014 & 4.4 & -3012 & -74.8 & -4.8 \\
\hline Total & 23027 & 100.0 & 23027 & 100.0 & & & \\
\hline
\end{tabular}




\section{Discusión}

El análisis de los datos de la matriz de transición reveló que alrededor del $70 \%$ del área de estudio ha sufrido modificaciones en casi todas las coberturas de suelo identificadas dentro del área de estudio. Las coberturas que experimentaron mayores cambios corresponden a pastizales permanentes, Fec 20 - $70 \%$, cultivos arbóreos y viñedos de secano, cultivos de regadío, Fcc $<20 \%$, Fcc $>70 \%$ y el improductivo. En efecto, los pastizales permanentes aumentaron casi 6 veces registrada en el año 1984. En los cultivos arbóreos y viñedos de secano, cultivos de regadío, Fcc $<20 \%$ e improductivo aun cuando el cambio neto fue negativo, la mayor parte de los suelos incorporados a esta actividad se atribuyen a la habilitación de pastizales permanentes, arbustos y matorral y cultivos herbáceos de secano. La Matriz nos indica que el abandono de los cultivos de regadío y cultivos arbóreos y viñedos de secano dio paso a una gran proporción de pastizales permanentes, arbustos y matorral Fcc $20-70 \%$. En tanto que el Fcc $<20 \%$ registró una pérdida de casi el $90 \%$ en 29 años (Tab. 2).

Tabla 2. Matriz de transición. La suma de las columnas y filas corresponde al área total de cada cobertura en el año 1984 y 2013.

\begin{tabular}{|c|c|c|c|c|c|c|c|c|c|c|c|}
\hline \multirow{12}{*}{ 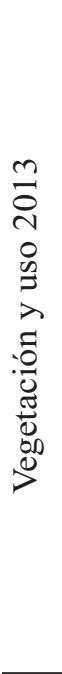 } & & \multicolumn{9}{|c|}{ Vegetación y uso 1984} & \multirow[b]{2}{*}{ Total } \\
\hline & & 1 & 2 & 3 & 4 & 5 & 6 & 7 & 8 & 9 & \\
\hline & 1 & 162 & 0 & 0 & 112 & 154 & 47 & 63 & 18 & 91 & 647 \\
\hline & 2 & 1 & 0 & 0 & 3 & 0 & 0 & 4 & 0 & 0 & 8 \\
\hline & 3 & 1 & 0 & 5 & 4 & 0 & 0 & 0 & 0 & 0 & 9 \\
\hline & 4 & 163 & 69 & 162 & 1667 & 659 & 209 & 698 & 57 & 46 & 3731 \\
\hline & 5 & 511 & 34 & 173 & 122 & 3721 & 251 & 1987 & 355 & 874 & 8029 \\
\hline & & 690 & 0 & 75 & 682 & 1799 & 362 & 727 & 170 & 853 & 5358 \\
\hline & 7 & 90 & 4 & 12 & 120 & 52 & 13 & 65 & 5 & 101 & 461 \\
\hline & 8 & 251 & 0 & 5 & 318 & 651 & 44 & 369 & 636 & 1497 & 3770 \\
\hline & 9 & 18 & 0 & 9 & 36 & 92 & 9 & 30 & 255 & 564 & 1014 \\
\hline & Total & 1887 & 108 & 441 & 3064 & 7128 & 935 & 3944 & 1495 & 4026 & 23027 \\
\hline
\end{tabular}

Nota: 1) Improductivo, 2) Cultivos de regadío, 3) Cultivos arbóreos y viñedos de secano, 4) Cultivos herbáceos de secano, 5) Arbustos y matorral, 6) Pastizales permanentes, 7) Fcc <20\%, 8) Fcc $20-70 \%$, y 9) Fcc $>70 \%$.

Las principales fuerzas transformadoras del paisaje aquí analizado no difieren de las que otros autores ya han identificado en la cuenca del Jarama, principalmente a la forestación espontanea, (García Ruiz et al., 1996). Otros estudios por el contrario, se centran en la determinación, del efecto que presenta el tipo de cubierta vegetal en componentes concretos del ciclo hidrológico (Llorens ,1997 y Llorens et al., 2003).

De acuerdo a los resultados, los cambios más importantes se deben a la actividad agropecuaria y forestal (Fcc 20 - 70 \% de cubierta y pastizales permanentes). Distin- 
tos autores han señalado que la transformación inicial del paisaje fue producto del éxodo a las comunidades urbanas y la habilitación de campos agrícolas a expensas de la tala o quema de la vegetación nativa que cubría gran parte del territorio del Jarama (Shakesby y Doerr, 2006).

\section{Conclusiones}

Los resultados de este estudio confirman que las principales transformaciones del paisaje son consecuencia del desarrollo de la actividad del arbolado con cabida Fcc 20 - $70 \%$ y pastizales permanentes con un balance neto positivo de $152.24 \%$ y un $473.11 \%$, respectivamente; no obstante también arrojan resultados netos negativos para la actividad de improductivo con $65.71 \%$, cultivos arbóreos y viñedos de secano con $97.86 \%$, erial a pastos Fcc $<20 \%$ con $88.30 \%$, arbolado con cabida Fcc $>70 \%$ con un $74.81 \%$ y los cultivos de regadío con un $92.82 \%$. Asimismo solo los cultivos herbáceos de secano presentan un leve movimiento positivo de $1.81 \%$ de balance neto.

\section{Agradecimientos}

La contribución del segundo autor ha sido posible gracias a la Beca Mixta predoctoral otorgada por el Consejo Nacional de Ciencia y Tecnología de México (CONACYT) y la colaboración directa del Departamento de Ingeniería y Gestión Forestal y Ambiental de la Universidad Politécnica de Madrid, a través de su titular Dr. José Luis García Rodríguez.

\section{Bibliografía}

Aguayo M, Wiegand T., G, Azócar, K., Wiegand y Vega C., 2007. Revealing the driving forces of mid-cities urban growth patterns using spatial modeling: a case study of Los Ángeles, Chile. Ecol Soc 12: 13.

Baret, F. y G. Guyot.1991. Potential and limits of vegetation indices for LA1 and APAR assessment. Remote Sens. Environ., 35: 161-1 73.

Berlanga-Robles C.A., Ruiz-Luna A., 2002. Land use and mapping and change detection in the coastal zone of northwest Mexico using remote sensing techniques. J. Coastal Res. 18(3): 514-522.

Bernabe V., Riesco J.A., Giménez M.C. y García J.L. 2014. Using remote sensing for monitoring of gully erosion progress in Jarama river basin. Guadalajara, Spain. In Proceedings of Brasov Symposium.

Boix-Fayos C., Barbera G.G.; Lopez-Bermúdez F. y Castillo V.M., 2007. Effects of check.dams, reforestation and land uses changes on river channel morphology: Case study of the Rogativa catchment (Murcia, Spain). Geomorphology 91:103-123. 
Brovkin V., Sitch S., Von Bloh W., Claussen M., Bauer E. et al., 2004. Role of land cover changes for atmospheric $\mathrm{CO} 2$ increase and climate change during the last 150 years. Glob. Change Biol. 10: 1253-1266.

Carreiras J; Shimabukuro, y Pereira, J., 2002. Fraction images derived from SPOT-4 VEGETATION data to assess land-cover change over the State of Mato Grosso, Brazil. Int. J. Remote Sens. 23(23):4979-83.

Chase T.N., Pielke R.A., Kittel T.G.F., Neman I R.R. y Running S.W., 2000. Simulated impacts of historical land cover changes on global climate in northern winter. Clim. Dynam. 16: 93-105.

Claussen M, Brovkin V. y Ganopolsk I., 2001. Biogeophysical versus biogeochemical feedbacks of large-scale land cover change. Geophys. Res. Lett. 28: 1011-1014.

Chiesi, M; Maselli, F; Bindi, M; Fibbi, L; Cherubini, P; Arlotta, E; Tirone, G; Matteucci, G; Seufert, G. ,2005 Modelling carbon budget of Mediterranean forests using ground and remote sensing measurements. Agric. Forest Meteorol., 135:22-34.

Defries R.S., Bounoua L.y Collatz G.J., 2002. Human modification of the landscape and Surface climate in the next fifty years. Glob. Change Biol. 8: 438-458.

Dobson J, Bright E, Ferguson R, Field D, Wood L, Haddad K, Iredale III H., Jensen J, Klemas V, Orth R, Thomas J.,1995. NOAA's coastal change analysis program, guidance for regional implementation. National Marine Fisheries Service, National Oceanic and Atmospheric Administration Technical Report 123, USA.

Echeverría C, Coomes D., Salas J., Rey Benayas J.M., Lara A. et al., 2006. Rapid deforestation and fragmentation of Chilean temperate forests. Biol. Conserv. 130: 481-494.

F.A.O., 1996. Forest resources assessment 1990. Survey of tropical forest cover and study change process. ome $\mathrm{N}^{\circ} 26.152 \mathrm{pp}$.

Foody. G. M. y Hill. R. A., 1996. Classification of (eds.), tropical forests classes from Landsat TM data, Int. J. Remote Sens., 17 2353-2367.

Foley JA, Defries R., Asner G.P., Barford G.C., Bonan G. et al., 2005. Global consequences of land use. Science 309: 570-574.

Garcìa-Ruiz, J.M.; Lasanta, T.; Ruiz-Flaño, P.; Ortigosa, L.; White, S.; Gonzalez, C. y Marti, C., 1996. Land-use change and sustainable development in mountain areas: a case study in the Spanish Pyrenees. Landscape ecol. 11(5): 267-277.

Houghton Ra, JL Hackler y Lawrence K., 1999. The US carbon Budget Contributions from landuse change. Science 285: 574-578.

Jensen J.R., Cowen D.J., Althausen J.D., Narumalani S., Weatherbee O. ,1998. An evaluation of coastwatch change detection protocol in South Carolina. En: Lunetta RS, Elvidge D (eds) Remote sensing change detection. Evironmental monitoring methods and applications. Ann Arbor Press, USA. 75-88 pp.

Lambin E.F., Turner B.L., Geist H.J., Agbola S.B., Angelsen A. et al., 2001. The causes of land-use and land-cover change: moving beyond the myths. Global Environ. Change. 11: 261-269.

Lambin, E. F., Baulies N., Bockstael G., Fisher T., Krug R., Lemmans E. F., Moran R. R., Rindfuss Y., Sato D., Skole B. L., Turner II and Vogel C.,1999. Land use and land cover change implementation strategy, IGBP report, 48, IHDP, report 10, Estocolmo.

Lhermitte, S; Verbesselt, J.; Jonckheere, I.; Nackaerts, K.; Van Aardt, J.A.N.; Verstraeten, W.W.; Coppin, P. , 2008. Hierarchical image segmentation based on similarity of NDVI time series. Remote Sens. Environ, 112:506-521 
López E., Bocco G., Mendoza M. y Duhau E., 2001. Predicting land-cover and land-use change in the urban fringe - A case in Morelia city, Mexico. Landscape Urban Plan. 55: 271-285.

Llorens, P., 1997. Rainfall interception by a Pinus sylvestris forest pach overgrow in a Mediterranean mountainous abandoned area II. Assessment of applicability of Gash`s analytical model. J. Hydrol. 199:346-359.

Llorens P.; Latron, J y Oliveras, I., 2003. Modelizacion del efecto del cambio global en la hidrología superficial. Ejemplo de aplicación de una cuenca Mediterránea de montaña. In: Procceding 3a Asamblea Hispano-Portuguesa de Geodesia y Geofisica. III: 1679-1681.

Matson PA, Parton W.J., Power A.G. y Swift M.J., 1997. Agricultural intensification and ecosystem properties. Science 277: 504-509.

Mas J.F.,1999. Monitoring land-cover changes: a comparison of change detection techniques Int. J. Remote Sens. 20(1): 139-152.

Moulin, S; Zurita, R; Guérif, M; Baret, F. 2003. Characterizing the spatial and temporal variability of biophysical variables of a wheat crop using hyper-spectral measurements. En: International Geoscience and remote sensing Symposimum, July 2003. Toulouse, Francia.

Pimm S.L. y Raven P., 2000. Biodiversity - Extinction by numbers. Nature 403: 843-845.

Ramsey III E.W., Nelson G.A., Sapkota S.K. 2001. Coastal change analysis program implemented in Louisiana. J. Coastal Res. 17(1): 53-71.

Sala O.E., Chapin F.S., Armesto J.J., Berlow E., Bloomfield J. et al., 2000. Biodiversity - Global biodiversity scenarios for the year 2100. Science 287: 1770- 1774.

Shakesby, R. A., y Doerr, S.H., 2006. Wilfire as an hydrological and geomorphologycal

Spanner, M.A., Pierte L.L., Peterson D. L. y Running S. W. 1990. Remote sensing of temperate coniferous forest leaf area index the influence of canopy closure, understory vegetation and background reflectance. Int. J. Remote Sens., 11 95-1 12.

Tarnavsky, E.; Garrigues, S.; Brown, M.E., 2008. Multiscale geostatistical analysis of AVHRR, SPOT-VGT, and MODIS global NDVI products. Remote Sens. Environ, 112:535-49.

Vitousek P.M., Mooney H.A., Lubchenco J. y Melillo J.M., 1997. Human domination of Earth's ecosystems. Science 277: 494-499. 